\title{
Timing for the CMS Phase-II Upgrade
}

\author{
Pablo Martinez Ruiz del Arbol ${ }^{a, *}$ \\ ${ }^{a}$ Instituto de Fisica de Cantabria, \\ Avda. Los Castros s/n, Santander, Spain \\ E-mail: parbol@ifca.unican.es
}

The Compact Muon Solenoid (CMS) detector at the Large Hadron Collider (LHC) is undertaking a Phase II upgrade program to face the harsh conditions imposed by the High Luminosity LHC (HL-LHC). This program comprises the installation of a new timing layer to measure the time of passage of minimum ionizing particles (MIP) with a time resolution of 30 ps. This MIP Timing Detector (MTD) will be composed of a central barrel timing layer (BTL) covering a pseudorapidity range up to 1.4, and a endcap timing layer (ETL) reaching a pseudorapidity of 3. The BTL will consist of LYSO:CE crystals while the ETL will be instrumented with Low Gain Avalanche Detectors (LGAD). The time information of the tracks will improve the rejection of spurious tracks and vertices, will allow to perform particle identification based on the time-of-flight and will bring unique physics opportunities in interesting signatures such as those including long-lived particles.

The Eighth Annual Conference on Large Hadron Collider Physics-LHCP2020

25-30 May, 2020

online

\footnotetext{
${ }^{*}$ Speaker
} 


\section{Introduction}

The High Luminosity LHC (HL-LHC) [1] will impose challenging conditions to the LHC experiments. In particular, the number of spurious vertices (pile-up) will scale up to 140-200 in every brunch crossing. Such a busy environment will derive into a large detector occupancy, deteriorating the performance of the reconstruction algorithms and having a strong impact on the physics programs of the experiments. The Compact Muon Solenoid (CMS) [2] detector is undertaking a Phase II upgrade program to mitigate the effect of the conditions imposed by the HL-LHC. The MIP Timing Detector (MTD) [3] is a timing layer that will measure the time of passage of minimim ionizing particles (MIP) with a resolution in the range 30-50 ps. The MTD consists of a central barrel timing layer (BTL) and the endcap timing layer (ETL), composed of two endcaps, providing hermetic coverage up to a pseudorapidity of 3 . The information provided by this system will allow to assign a timestamp to the tracks, helping with the rejection of pile-up vertices, improving the reconstruction of the physics objects and also bringing unique physics opportunities.
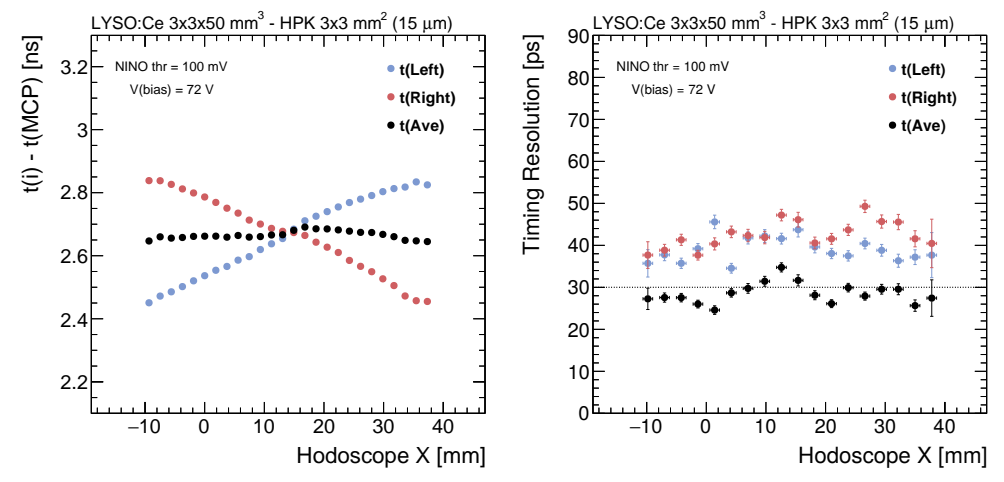

Figure 1: Time response (left) and time resolution (right) as a function of the longitudinal coordinate of the crystal.

\section{The Barrel Timing Layer}

The BTL is composed by a total of 166000 LYSO:Ce crystals [4], read out with two SiPMs at the two ends. Each crystal unit has a length of about $57 \mathrm{~mm}$ and a section of $3 \times 3 \mathrm{~mm}^{2}$ and are organized in modules of $1 \times 16$ crystals, arranged in read-out units of $3 \times 8$ modules and finally located in BTL trays. The BTL trays are installed at the inner side of the Tracker support tube, close to the last Tracker layer. The SiPMs attached to the crystals are readout with dedicated ASICs (TOFHIR), reading 32 channels each. The choice of LYSO:Ce crystals was based on their excellent radiation tolerance, high light yield, and fast rise and decay times. The time resolution of the crystals is expected to be $35 \mathrm{ps}$ at the beginning of operation and about 50-60 ps after an integrated luminosity of $3000 \mathrm{fb}^{-} 1$. The performance of the crystals has been measured in test-beams, showing a very uniform time response along the crystal lenght as it is shown in Fig.1 (left), and a excellent time resolution, Fig.1 (right), reaching a resolution of 30 ps once the information of the two sides of the crystal is combined. 


\section{The Endcap Timing Layer}

The ETL will use Low Gain Avalanche Diodes (LGADs) [5] as detection technology. The LGADs have a sufficient radiation tolerance to cope with the fluence expected in the endcap regions $\left(2 \times 10^{15} \mathrm{neq} / \mathrm{cm}^{2}\right)$ and also offer a very good timing resolution, with $30 \mathrm{ps}$ at the beginning of the operation and 45 ps at the end of the lifetime. Each of the sides of the ETL is composed of two disks, instrumented on both sides each, in such a way that two hits are expected for most of the tracks. The LGAD pixels will have a section of $1.3 \times 1.3 \mathrm{~mm}^{2}$ in order to keep the capacitance low. They are organized in arrays of 16x32 pixels, and are readout with two ETL ASICs (known as ETROC). The performance of the sensors have been measured in test-beams showing and excellent efficiency (>99\%) and uniform time resolution.

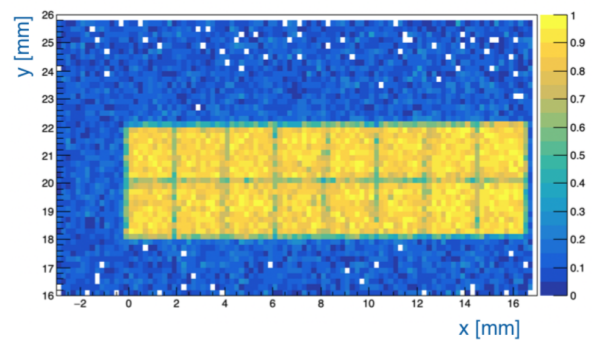

Figure 2: Efficiency of a 16 pad sensors measured in an FNAL beam test with $120 \mathrm{GeV}$ protons.

\section{Performance of physics objects and physics analysis}

The inclusion of time information will impact the CMS reconstruction in many ways. First of all, the time information of the tracks will allow to reject pile-up tracks with timestamps not compatible with the time associated to the primary vertex. This capability of cleaning the events from spurious tracks will also have an impact on the estimation of the lepton isolation, the b-tagging algorithms, or the resolution of the transverse missing momentum. In addition, the time information allows to implement a particle identification based on the Time-Of-Flight of the particles.

All the improvements in the reconstruction of the physics objects will finally impact the physics analysis. For instance, Fig. 3 (left) shows how the improved photon reconstruction increases the signal efficiency more than $20 \%$ in the double Higgs production analysis where one of the Higgs bosons decays into b-jets and the other into photons. The MTD information also provides handles to improve and extend the searches for new physics in difficult topologies such as those including long-lived particles or heavy stable charged particles. Figure 3 (right) shows an example of a search for a Gauge Mediated Supersymmetry Breaking (GMSB) model in which a long-lived neutralino produces a couple of displaced leptons in the final state, in addition to a Gravitino. The time information allows to reconstruct the mass of the neutralino under the assumption that the gravitino is massless. 

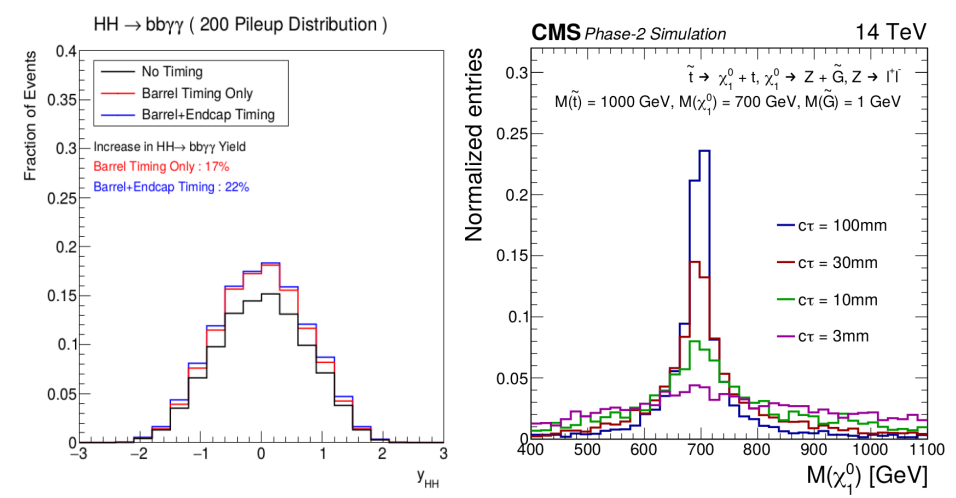

Figure 3: Fraction of events as a function of the rapidity in the context of the HH analysis in a final state with two b-jets and two photons (left), and reconstructed mass of the long-lived neutralino in a GMSB SUSY model with displaced leptons in the final state (right).

\section{Conclusions}

The CMS Phase II Upgrade program of CMS includes a MIP Timing Detector (MTD) that will measure the time of passage of charged particles with a time resolution of 30-50 ps. The MTD is composed of a central barrel timing layer (BTL), using LYSO:Ce crystals as detection technology, and the endcap timing layer (ETL), in the forward regions, and using Low Gain Avalanche Detectors (LGADs). The MTD information will allow to improve the rejection of spurious tracks in the busy environment of the HL-LHC and will also help to improve the performance of the physics objects reconstruction. All these developments will contribute to improve the Physics program of CMS and will bring unique physics potential in specific areas such as the searches for long-lived particles or heavy stable charged particles.

\section{References}

[1] Apollinari, G. and Bruning, O. and Nakamoto, T. and Rossi, Lucio. High Luminosity Large Hadron Collider HL-LHC. CERN Yellow Rep. 5 1-19, 2015. 10.5170/CERN-2015-005.1

[2] Chatrchyan, S. and others. The CMS experiment at the CERN LHC. JINST, 3 S08004, 2008, 10.1088/1748-0221/3/08/S08004

[3] Chatrchyan, S. and others. A MIP Timing Detector for the CMS Phase 2 Upgrade. CERNLHCC-2019-003, CMS-TDR-020

[4] A. Benaglia, S. Gundacker, P.Lecoq, M.T.Lucchini, A.Para, K.Pauwels, E.Auffray. Detection of high energy muons with sub-20 ps timing resolution using L(Y)SO crystals and SiPM readout. Nucl. Instrum. Meth. A 830 (2016), doi:10.1016/j.nima.2016.05.030

[5] G. Pellegrini et al. Technology developments and first measurements of Low Gain Avalanche Detectors (LGAD) for high energy physics applications. Nucl. Instrum. Meth. A 765 (2014) 12, doi:10.1016/j.nima.2014.06.008 